\title{
Local spin dynamics of iron oxide magnetic nanoparticles dispersed in different solvents with variable size and shape: A ${ }^{\mathbf{H}}$ NMR study
}

M. Basini, T. Orlando, P. Arosio, M. F. Casula, D. Espa, S. Murgia, C. Sangregorio, C. Innocenti, and A. Lascialfari

Citation: The Journal of Chemical Physics 146, 034703 (2017); doi: 10.1063/1.4973979

View online: http://dx.doi.org/10.1063/1.4973979

View Table of Contents: http://aip.scitation.org/toc/jcp/146/3

Published by the American Institute of Physics

\section{Articles you may be interested in}

On the magnetic anisotropy and nuclear relaxivity effects of $\mathrm{Co}$ and $\mathrm{Ni}$ doping in iron oxide nanoparticles Journal of Applied Physics 119, 134301 (2016); 10.1063/1.4945026

Hydrogen induced contrasting modes of initial nucleations of graphene on transition metal surfaces The Journal of Chemical Physics 146, 034704 (2017); 10.1063/1.4974178

Relaxation dynamics of a multihierarchical polymer network

The Journal of Chemical Physics 146, 034902 (2017); 10.1063/1.4973936

Identifying two regimes of slip of simple fluids over smooth surfaces with weak and strong wall-fluid interaction energies

The Journal of Chemical Physics 146, 034701 (2017); 10.1063/1.4973640

Comparative investigation of pure and mixed rare gas atoms on coronene molecules

The Journal of Chemical Physics 146, 034302 (2017); 10.1063/1.4973890

Simulations of polymer brushes with charged end monomers under external electric fields

The Journal of Chemical Physics 146, 034901 (2017); 10.1063/1.4973932

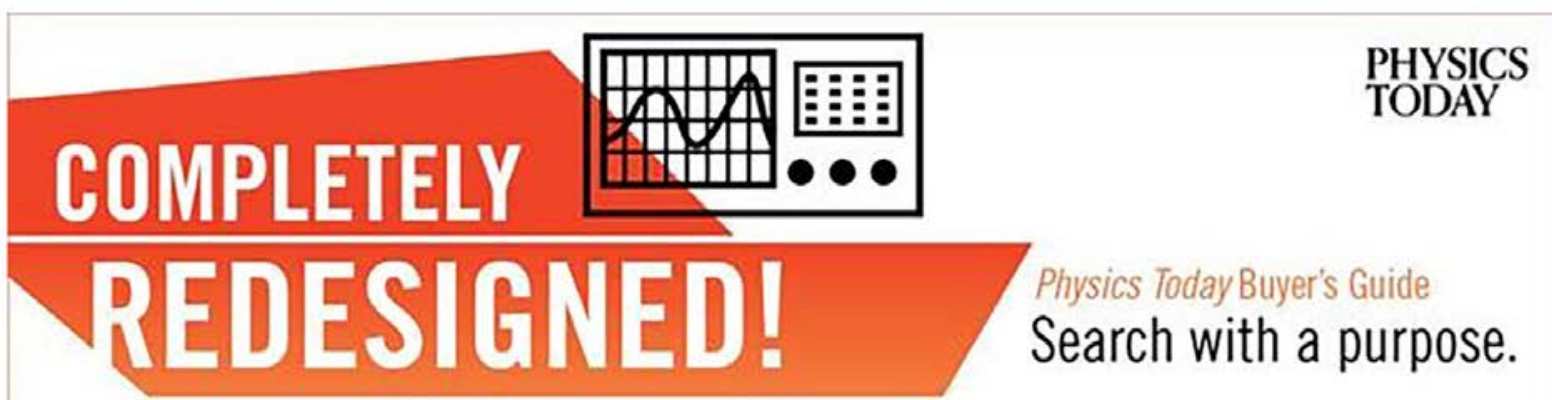




\title{
Local spin dynamics of iron oxide magnetic nanoparticles dispersed in different solvents with variable size and shape: ${ }^{1}{ }^{1} \mathbf{H}$ NMR study
}

\author{
M. Basini, ${ }^{1}$ T. Orlando, ${ }^{2}$ P. Arosio, ${ }^{1}$ M. F. Casula, ${ }^{3}$ D. Espa,${ }^{3}$ S. Murgia, ${ }^{3}$ \\ C. Sangregorio, ${ }^{4}$ C. Innocenti, ${ }^{5}$ and A. Lascialfari ${ }^{1,6}$ \\ ${ }^{1}$ Dipartimento di Fisica and INSTM, Università degli Studi di Milano, Milano, Italy \\ ${ }^{2}$ EPR Research Group, Max Planck Institute for Biophysical Chemistry, Göttingen, Germany \\ ${ }^{3}$ Dipartimento di Scienze Chimiche e Geologiche and INSTM, Università di Cagliari, Monserrato, Italy \\ ${ }^{4}$ ICCOM-CNR and INSTM, Sesto Fiorentino, Italy \\ ${ }^{5}$ Dipartimento di Chimica and INSTM, Università degli studi di Firenze, Sesto Fiorentino, Italy \\ ${ }^{6}$ Istituto di Nano scienze, CNR-S3, Modena, Italy
}

(Received 5 August 2016; accepted 30 December 2016; published online 18 January 2017)

\begin{abstract}
Colloidal magnetic nanoparticles (MNPs) based on a nearly monodisperse iron oxide core and capped by oleic acid have been used as model systems for investigating the superparamagnetic spin dynamics by means of magnetometry measurements and nuclear magnetic resonance $\left({ }^{1} \mathrm{H}\right.$ NMR) relaxometry. The key magnetic properties (saturation magnetization, coercive field, and frequency dependent "blocking" temperature) of MNPs with different core size $(3.5 \mathrm{~nm}, 8.5 \mathrm{~nm}$, and $17.5 \mathrm{~nm})$, shape (spherical and cubic), and dispersant (hexane and water-based formulation) have been determined. ${ }^{1} \mathrm{H}$ NMR dispersion profiles obtained by measuring the $r_{1}$ (longitudinal) and $r_{2}$ (transverse) nuclear relaxivities in the frequency range $0.01-60 \mathrm{MHz}$ confirmed that in all samples the physical mechanisms that drive the nuclear relaxation are the Néel reversal at low temperature and the Curie relaxation at high frequency. The magnetization reversal time at room temperature extracted from the fitting of NMR data falls in the typical range of superparamagnetic systems $\left(10^{-9}-10^{-10} \mathrm{~s}\right)$. Furthermore, from the distance of minimum approach we could conclude that water molecules do not arrive in close vicinity of the magnetic core. Our findings contribute to elucidate the local spin dynamics mechanisms in colloidal superparamagnetic nanoparticles which are useful in biomedical application as, e.g., contrast agents for magnetic resonance imaging. Published by AIP Publishing. [http://dx.doi.org/10.1063/1.4973979]
\end{abstract}

\section{INTRODUCTION}

In the last twenty years, superparamagnetic (SPM) iron oxide nanoparticles have captured the interest of multidisciplinary research activities and, recently, much attention has been devoted to understand the biomedical potential of theranostic systems based on magnetic nanoparticles (MNPs). The number of applications of magnetism to nanomedicine, including the well-known Magnetic Resonance Imaging (MRI), Magnetic Fluid Hyperthermia (MFH), cell separation, biosensing, and cellular functionalities study, is rapidly growing. ${ }^{1-8}$ Though MNPs with different morphologies have been developed for biomedical applications, a full comprehension of the physical mechanisms and particularly the spin dynamics in the presence of biological media and related to their size, shape, coating, and dispersant has not been achieved yet. On the other hand, these morphological characteristics are crucial to determine their efficacy as contrast agents (CAs) in MR images quantified by an increase of the nuclear relaxation rate (referred to as nuclear relaxivity) of the ${ }^{1} \mathrm{H}$ nuclei contained in the human body. ${ }^{9}$ The achievement of a higher nuclear relaxivity leads to a better contrast in the images and allows us also to envisage a reduction of the maximum dose injectable in living beings, a crucial issue for their safety. Both applied and fundamental ${ }^{10-13}$ current researches on superparamagnetic ferrite-based CAs are therefore often devoted to understand the physical mechanisms of enhancement of the nuclear relaxation rates and to develop physical models able to predict the design of MNPs with optimized features. Within this framework, it is crucial to study the influence of the main MNP microscopic characteristics like size, shape, and composition of the magnetic core, surface coating, and the dispersant on the magnetic properties and on the nuclear magnetic resonance (NMR) relaxation rates. This task is very hard to achieve because of the mutual dependence of the above parameters and the data in the literature are very often difficult to compare.

With the present work we aim at contributing to clarify the influence of some MNPs properties, like size, shape, and dispersant on the nuclear longitudinal $\left(\mathrm{r}_{1}\right)$ and transversal $\left(\mathrm{r}_{2}\right)$ relaxivities and on the dynamical magnetic properties of colloidal suspensions. For this purpose, we performed ${ }^{1} \mathrm{H}$ nuclear magnetic resonance dispersion (NMR-D) and DC/AC magnetization measurements on iron-oxide based nanocrystals synthesized by high temperature colloidal routes. To study the role of the core size, we investigated nearly spherical MNPs with three different sizes, whereas to take into account the effect of the shape, MNPs with the same volume and different morphology (spherical and cubic) were considered. Moreover, in order to study the effect of the dispersing media on the contrast efficiency, the same sample was suspended either in an organic non-polar solvent (hexane) or in water, without removing the ligand directly attached at the surface of the inorganic core. 
The AC/DC magnetic data allowed us to evaluate the energy barrier distribution as a function of the external magnetic field and the spin correlation time (Néel time). The same data joint to the $r_{1}$ and $r_{2}$ vs frequency ( $v$ ) profiles offered the opportunity to evaluate the reliability of the wellknown model developed by Roch et al. ${ }^{14,15}$ for superparamagnetic relaxation. In particular we analyzed simultaneously the $r_{1}(v)$ and $r_{2}(v)$ experimental curves over a wide frequency $(0.01<v<120 \mathrm{MHz})$ range, a method that on variable size maghemite-based systems has not still been used in the literature. Finally, our data were also used to test a recently proposed universal scaling law by Vuong et al. ${ }^{16}$ which describes the transversal relaxivity of iron oxide MNPs in different dynamic regimes.

\section{EXPERIMENTAL SECTION: MATERIALS AND METHODS}

\section{A. Experimental techniques}

In order to discriminate the iron oxide phase of the magnetic nanoparticles X-Ray diffraction (XRD), wide angle patterns were recorded using $\mathrm{Cu} \mathrm{Ka}$ radiation on a Panalytical Empyrean diffractometer equipped with a focusing mirror on the incident beam and an $\mathrm{X}^{\prime}$ Celerator linear detector. The scans were collected in Bragg-Brentano geometry with a graphite monochromator on the diffracted beam and the $\mathrm{X}^{\prime}$ Celerator linear detector. The dispersions were deposited on a lowbackground Si substrate and let to evaporate under ambient conditions.

The size and shape of the magnetic core of the MNPs were determined by transmission electron microscopy (TEM) images, recorded on a Hitachi H7000 microscope, operating at $125 \mathrm{kV}$, equipped with a $\mathrm{W}$ thermionic electron source. Prior to observation, the diluted samples were deposited on a carbon-coated copper grid and let to evaporate under ambient conditions.

The hydrodynamic size distribution of the disperse nanoparticles in the ferrofluids was determined by dynamic light scattering (DLS) measurements, performed on a Malvern Zetasizer Nano-ZSP provided with a laser of $633 \mathrm{~nm}$ wavelength. The measurements were collected using a quartz cuvette with $1 \mathrm{~mm}$ optical path length. The intensity averaged diameter values were taken into account.

Chemical composition was determined by inductively coupled plasma atomic emission spectrometry (ICP-AES) using a Varian Liberty 200 spectrophotometer. Solutions for Fe analysis content were prepared by dissolving $0.5 \mathrm{ml}$ of each suspension in a $\mathrm{HCl}$ and $\mathrm{HNO}_{3}$ 3:1 mixture until complete dissolution of the nanocrystals and diluting to $100 \mathrm{ml}$. The Fe analysis provided the value of the metal concentration in suspension for the relaxometric studies.

Thermal gravimetry (TG) and differential thermal analysis (DTA) were carried out using a Mettler-Toledo TG/SDTA 851 in the range $25-1000{ }^{\circ} \mathrm{C}$ under oxygen flow (heating rate $=10^{\circ} \mathrm{C} / \mathrm{min}$, flow rate $=50 \mathrm{ml} / \mathrm{min}$ ). Thermograms were used to quantitatively assess the amount of oleic acid bound to the nanoparticle surface in order to normalize the magnetic data to the mass of the inorganic core.
DC magnetization and AC magnetic susceptibility have been recorded on dry powder samples by a Superconducting Quantum Interference Device (SQUID) magnetometer (MPMS-XL7 Quantum Design). The hysteresis curves have been collected as a function of the magnetic field (up to $\pm 5 \mathrm{~T}$ ) at different temperatures ( $300 \mathrm{~K}$ and $5 \mathrm{~K}$ ). Zero-FieldCooled (ZFC) and Field-Cooled (FC) magnetization curves have been recorded as a function of temperature $(5-300 \mathrm{~K})$ at different static magnetic fields (ranging from 0.005 to $0.3 \mathrm{~T}$ ) after cooling the sample in the absence (ZFC) or in the presence (FC) of the measuring field. AC magnetic susceptibility data of colloidal solutions of the different samples have been collected as a function of temperature (5-300 K) and at different frequencies in the range $1-1000 \mathrm{~Hz}$.

The ${ }^{1} \mathrm{H}$ nuclear magnetic resonance (NMR) relaxometry profiles were collected at room temperature in the frequency range $10 \mathrm{kHz} \leq v \leq 120 \mathrm{MHz}$ by measuring the longitudinal and the transverse nuclear relaxation times $T_{1}$ and $T_{2}$ of colloidal solutions of the different samples. The NMR signal detection and generation were obtained by a Smartracer Stelar relaxometer in the range $10 \mathrm{kHz} \leq v \leq 9.5 \mathrm{MHz}$, a Stelar Spinmaster, and an Apollo-Tecmag Fourier Transformnuclear magnetic resonance (FT-NMR) spectrometer for $v \geq 9.5 \mathrm{MHz}$.

\section{B. Synthesis of superparamagnetic colloidal iron oxide nanoparticles}

Iron oxide MNPs were prepared according to surfactantmediated colloidal routes in high boiling organic solvents under nitrogen atmosphere, using Schlenk line procedures. A previous investigation ${ }^{21,22}$ demonstrated that such a surfactant mixture forms vesicles.

The preparation route, which was adapted from Ref. 35, relies on two steps including (i) the syntheses of the iron oxide precursor and (ii) its thermal decomposition in a hot solution containing surfactants. ${ }^{17,18}$ For all syntheses, as iron oxide precursor, an iron (III) oleate complex was used. The iron oleate complex was obtained by reacting under $\mathrm{N}_{2}$ flow $2.700 \mathrm{~g}$ of iron chloride (Sigma-Aldrich 97\%) and $9.125 \mathrm{~g}$ of sodium oleate (Sigma, $\geq 99 \%$ ) in $70 \mathrm{ml}$ of a mixture of anhydrous ethanol, distilled water, and hexane (Fluka, $\geq 99 \%$ ) in the volume ratios 2:1.5:3.5 and heating under stirring at $60{ }^{\circ} \mathrm{C}$ for $4 \mathrm{~h}$. The iron oleate complex is finally obtained as a dark brown gel by removal of the solvent mixture in rotavapor. In the second step, the iron oleate complex was transferred into a flask together with $32 \mathrm{ml}$ octadecene (Aldrich, 90\%) and $0.710 \mathrm{~g}$ oleic acid (Sigma-Aldrich, 99\%) connected to a condenser and heated under nitrogen atmosphere. While keeping fixed the amount of precursor, solvent, and surfactant, different heating conditions (heating ramp, final temperature, and hold at the final temperature) were used to obtain nanoparticles with different size and shape. In particular, the overall heating time and final temperature reached were as follows (see Table I for samples' reference): $4 \mathrm{~h} 30^{\prime} / 320^{\circ} \mathrm{C}$ for S17_Hex; $1 \mathrm{~h} 30^{\prime} / 320^{\circ} \mathrm{C}$ for S8_Hex; $5 \mathrm{~h} 30^{\prime} / 295^{\circ} \mathrm{C}$ for C8_Hex. Sample S3_Hex was obtained by using the same ratio among chemicals but using diphenyl ether (Reagent Plus ${ }^{\circledR}$ Aldrich, $\geq 99 \%$ ) instead of octadecene as the solvent. ${ }^{18,19}$ In this case, the reaction was carried out by heating up to $250{ }^{\circ} \mathrm{C}$ with an overall 
TABLE I. Summary of the dispersant and morphological features of the investigated colloidal nanoparticles: $\langle\mathrm{d}\rangle_{\mathrm{TEM}}$ and $\langle\mathrm{d}\rangle_{\mathrm{XRD}}$ are the average MNPs sizes as determined by TEM and XRD techniques, respectively; the maximum absolute error on $\langle\mathrm{d}\rangle_{\mathrm{TEM}}$ and $\langle\mathrm{d}\rangle_{\mathrm{XRD}}$ was 0.2 and $0.9 \mathrm{~nm}$, respectively, for all samples. *The values refer to the square edge and diagonal, respectively. ** The primary nanocrystals in sample S8_Wat are the same as in sample S8_Hex; the aggregation of the nanocrystals varies as a consequence of the exchange procedure as detailed in Sec. II B and supplementary material.

\begin{tabular}{lcccc}
\hline \hline Sample & Shape & Dispersant & $\left\langle d_{T E M}\right\rangle(\mathrm{nm})$ & $\left\langle d_{X R D}\right\rangle(\mathrm{nm})$ \\
\hline S3_Hex & Sphere & Hexane & 3.5 & 3.8 \\
S8_Hex & Sphere & Hexane & 8.5 & 7.6 \\
C8_Hex & Cube & Hexane & $7.1 / 8.1^{*}$ & 7.4 \\
S8_Wat & Sphere & Water & $8.5^{* *}$ & 9.2 \\
S17_Hex & Sphere & Hexane & 17.5 & n.a. \\
\hline \hline
\end{tabular}

ramp rate of $4 \mathrm{~h}$. All the samples were purified by repeating for 3 times the following procedure: precipitation of the nanoparticles by adding absolute ethanol and centrifugation, followed by redispersion in hexane. An additional purification step included magnetic separation and collection of the nanoparticles, removal of the surnatant, and redispersion in hexane, in which the MNPs are fully dispersible being coated by oleic acid on their surface. Sample S8_Hex was also transferred into an aqueous based dispersant by evaporating hexane and then adding a ternary system made out of monoolein, lauroylcholin, and water (mass ratio w/w\% 3.3, 0.3, and $96.4)^{20}$ and sonicating for $15 \mathrm{~min}$. The so-obtained sample was referred as S8_Wat.Monoolein (MO, 1-monooleoylglycerol, RYLO MG 90-glycerol monooleate; 98.1 wt. \% monoglyceride) was kindly provided by Danisco Ingredients, Brabrand, Denmark. Lauroylcholine chloride (LCh, >98\%) was from TCI Europe.

\section{RESULTS AND DISCUSSION}

\section{A. Morphological and structural characterization}

Bright field TEM images of the MNPs samples are shown in Figure 1, together with the corresponding size distribution histograms as obtained by statistical analysis of the images. The nanocrystals exhibit a well-defined morphology, being faceted and nearly monodispersed in size and shape. The particle size of the spherical samples increases from sample S3_Hex to sample S17_Hex, the average diameter being $3.5 \mathrm{~nm}$ (sample S3_Hex), $8.5 \mathrm{~nm}$ (sample S8_Hex and S8_Wat), and $17.5 \mathrm{~nm}$ (sample S17_Hex). The sample C8_Hex has a cubic shape with a diagonal of $8.1 \mathrm{~nm}$ (7.1 square edge). All samples are dispersed in hexane, except for S8_Wat, which is dispersed in an aqueous solution of monoolein (MO) and lauroylcholin (LCh). ${ }^{20}$ The main morphological features and the dispersant are summarized in Table I.

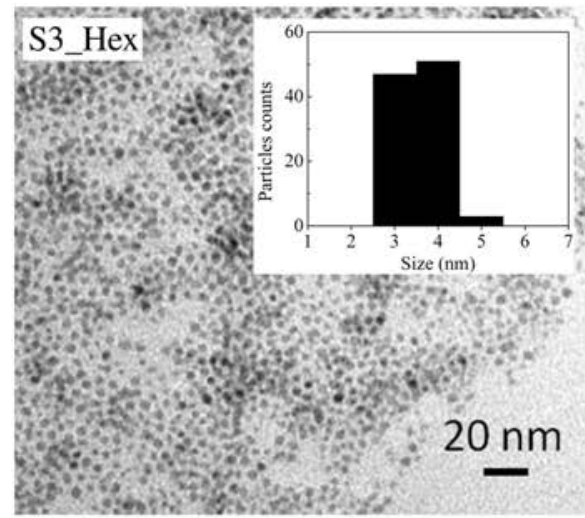

(a)

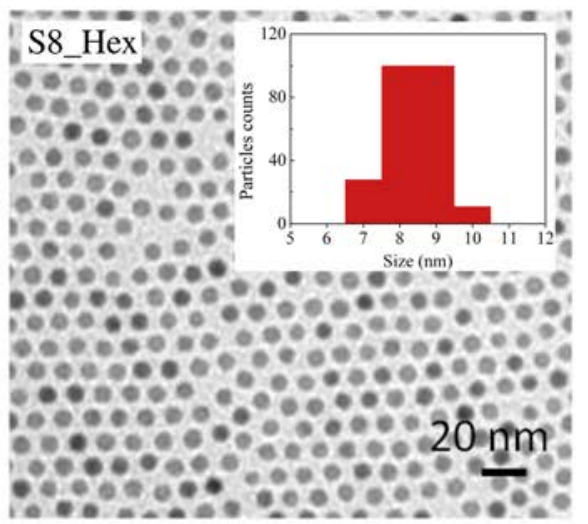

(b)

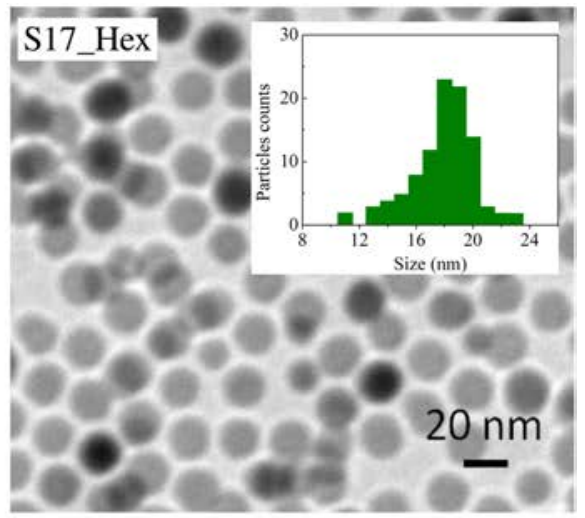

(c)

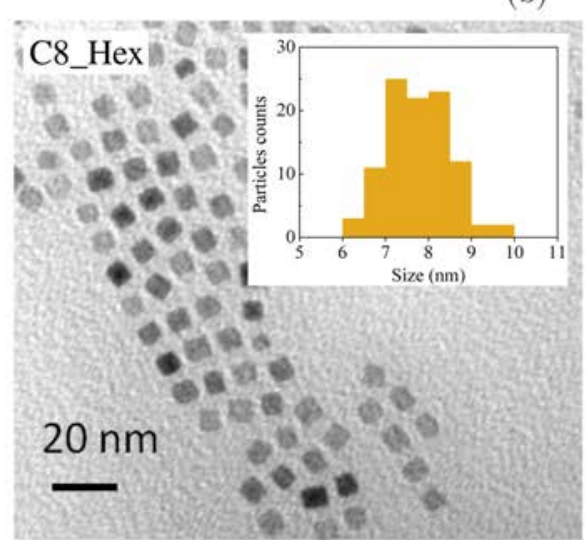

(d)
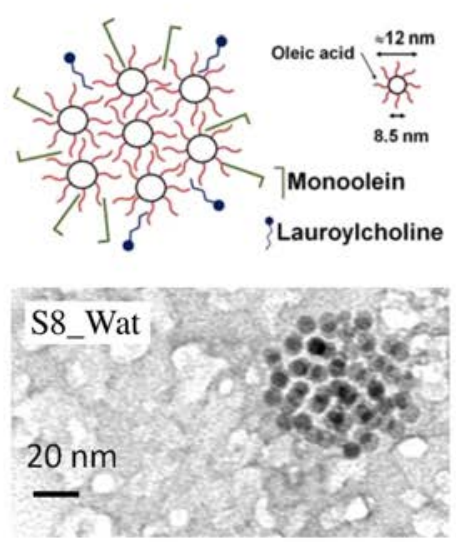

(e)

FIG. 1. TEM images of the iron oxide MNPs. Insets show the corresponding particle size distribution obtained by statistical analysis over $\sim 150$ particles. For the spherical particles ((a)-(c)) the diameter was considered, while for the cubic one (d) the diagonal was measured. The particle aggregation due to the dispersant effect in sample S8_Wat is shown in (e). 
The XRD patterns of the MNPs (reported in the supplementary material as Fig. S2) are quite similar for all samples. Mostly, they are dominated by broad reflections due to the presence of nanocrystalline iron oxide spinel phase. The observed patterns can be ascribed either to the $\gamma$ polymorph of the ferric oxide $\left(\gamma-\mathrm{Fe}_{2} \mathrm{O}_{3}\right.$, maghemite) or the mixed valence iron oxide $\left(\mathrm{Fe}_{3} \mathrm{O}_{4}\right.$ magnetite). In the case of sample $\mathrm{S} 17 \_\mathrm{Hex}$, a small contribution of $\mathrm{FeO}$ (wüstite) is also detected. The particles size, determined by the peak broadening, is in agreement with the TEM observation (for further details please refer to the supplementary material).

Since TEM and XRD give information mainly about the inorganic iron oxide core of the MNPs, dynamic light scattering (DLS) has been performed on colloidal suspensions in order to estimate the size of the oleic acid layer used as a capping agent. All samples present a narrow and symmetrical distribution of hydrodynamic diameters, whose average value, $\left\langle\mathrm{d}_{\mathrm{DLS}}\right\rangle$, is reported in Table IV.

Indeed, DLS experiments performed on sample S8_Wat confirmed the establishment of a quite polydispersed colloidal suspension characterized by an average diameter of $50 \mathrm{~nm}$ (see Table IV) and a $\zeta$-potential of $+84 \mathrm{mV}$. TEM investigation further supported this picture, evidencing the presence of MNPs aggregated in clusters together with isolated nanoparticles (see Fig. 1(e) and Fig. S1).

\section{B. Magnetic characterization}

\section{Hysteresis curves}

Figure 2 shows the magnetization curves $\mathrm{M}(\mathrm{H})$ recorded at $5 \mathrm{~K}$ on dried powder samples (except for the sample S8_Wat, which was measured in water dispersion) with different size, shape, and dispersant. The loops evidence an open hysteresis at this temperature, indicating that the particles are in a blocked spin state. On the other hand, the $\mathrm{M}(\mathrm{H})$ curves recorded at 300 $\mathrm{K}$ (data not reported) have no hysteretic behaviour, confirming that all the samples are in the SPM regime at room temperature. The values of coercive field $\mathrm{H}_{\mathrm{c}}$, saturation magnetization, $\mathrm{M}_{\mathrm{s}}$, at $5 \mathrm{~K}$ and $300 \mathrm{~K}$, and reduced remanence, $\mathrm{M}_{\mathrm{r}} / \mathrm{M}_{\mathrm{s}}$, are listed in Table II. For spherical-shaped MNPs in hexane suspensions (samples S3_Hex, S8_Hex, and S17_Hex) the coercive field, $\mathrm{H}_{\mathrm{c}}$, increases with the average size (Fig. 2(a)), as expected for single domain MNPs of small size. ${ }^{5}$ The large value for S17 $(0.1 \mathrm{~T})$ can be partially ascribed to enhanced anisotropy due to exchange-coupling between magnetite
TABLE II. Magnetic parameters of the investigated MNPs measured by DC magnetometry: coercive field $H_{c}$, reduced remanent magnetization, $M_{r} / M_{s}$, and saturation magnetization $\mathrm{M}_{\mathrm{s}}$.

\begin{tabular}{lrccc}
\hline \hline & $\mu_{0} \mathrm{H}_{\mathrm{c}}(\mathrm{mT})$ & $\frac{M_{r}}{M_{s}}$ & $\mathrm{M}_{\mathrm{S}}(5 \mathrm{~K})(\mathrm{emu} / \mathrm{g})$ & $\mathrm{M}_{\mathrm{s}}(300 \mathrm{~K})(\mathrm{emu} / \mathrm{g})$ \\
\hline S3_Hex & $2.4 \pm 0.4$ & 0.05 & $33 \pm 1$ & $25 \pm 1$ \\
S8_Hex & $48.5 \pm 2.0$ & 0.24 & $84 \pm 1$ & $42 \pm 1$ \\
C8_Hex & $26.9 \pm 0.9$ & 0.22 & $71 \pm 1$ & $45 \pm 1$ \\
S8_Wat & $30.5 \pm 1.0$ & 0.21 & $84 \pm 1$ & $45 \pm 1$ \\
S17_Hex & $142.0 \pm 6.3$ & 0.24 & $34 \pm 1$ & $32 \pm 1$ \\
\hline \hline
\end{tabular}

and wustite. Further experiment needed to validate this hypothesis, however, is beyond the purpose of this work. The $\mathrm{H}_{\mathrm{c}}$ variations upon shape and solvent are not very significant; a slight decrease is indeed observed from spherical (S8-Hex) to cubic-shaped MNPs (C8_Hex), as shown in Figure 2(b), and switching the dispersant from hexane to water (Figure 2(c)).

Samples S8_Hex, C8_Hex, and S8_Wat display similar value for $\mathrm{M}_{\mathrm{s}}$, suggesting that the shape and the dispersant do not sizeably affect $M_{\mathrm{s}}$. On the contrary, $\mathrm{M}_{\mathrm{s}}$ significantly decreases upon size reduction (sample S3_Hex), as expected for fine nanoparticles. ${ }^{23}$ The low $\mathrm{M}_{\mathrm{s}}$ of sample S17_Hex, despite its large size, is ascribed to the presence of wustite in addition to magnetite/maghemite, as suggested by XRD analysis.

\section{DC and AC magnetic measurements as a function of temperature}

The magnetic dynamics of SPM systems is usually explained in terms of the Néel or Vogel-Fulcher (VF) models, ${ }^{25,26}$ which describe the spin-blocking process controlled by the competition between the magnetic anisotropy energy, $\mathrm{E}_{\mathrm{B}}$, and the thermal energy, $k_{B} \mathrm{~T}$, in the absence (Néel) or in the presence (VF) of interparticle interactions. Referring to the more general VF model, the magnetization reversal time is given by $\tau_{N}=\tau_{0}\left[\exp \left(E_{B} / k_{B}\left(T-T_{0}\right)\right)\right]$, where $\tau_{0}$ is the attempt time and $\mathrm{T}_{0}$ is a phenomenological parameter whose value is proportional to the interparticle interaction. The VF law reduces to the Néel one when $T_{0}$ is negligible. Following these models, the temperature at which the Néel relaxation frequency, $\tau_{\mathrm{N}}{ }^{-1}$, equals the measuring angular frequency $\omega_{\mathrm{m}}$ (typical of the investigating experimental apparatus), identifies the so-called blocking temperature $T_{B}$, which thus depends on $\tau_{0}, \mathrm{E}_{\mathrm{B}}$, and $\mathrm{T}_{0}$. To obtain information on the (a)

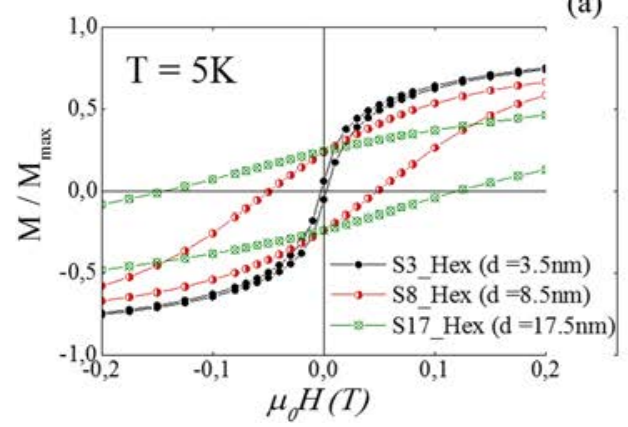

(b)

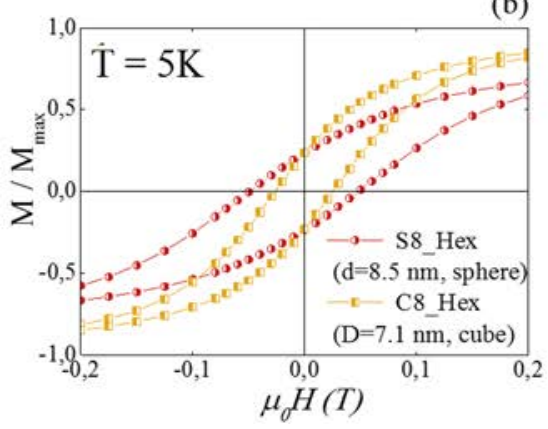

(c)

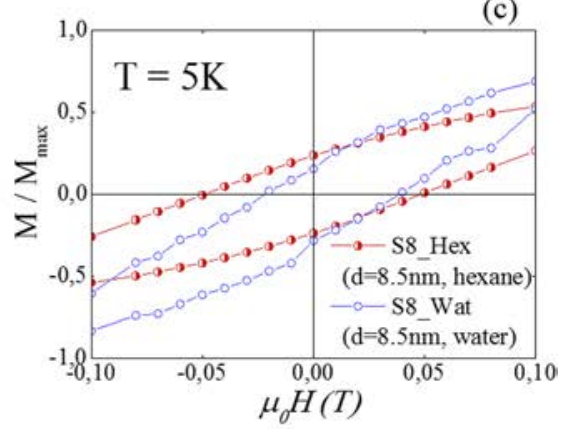

FIG. 2. $\mathrm{M}$ vs $\mathrm{H}$ (normalized to $\mathrm{M}_{\mathrm{s}}$ measured at $\mathrm{T}=5 \mathrm{~K}$ on powder samples with different: (a) size, (b) shape, and (c) dispersant. 
temperature dependence of the spin dynamics we therefore performed AC and DC magnetometry measurements, here below discussed.

a. DC magnetization. Figure 3 summarizes the zero field cooled (ZFC) and field cooled (FC) magnetization curves recorded by applying a $5 \mathrm{mT}$ static magnetic field for samples with different size, shape, and dispersant. The two curves superimpose in the region of temperature above the so-called irreversibility temperature $T_{\text {irr }}$, while the ZFC curve exhibits a maximum at $\mathrm{T}_{\max } \mathrm{DC}<\mathrm{T}_{\text {irr, }}$, which is generally identified as the simplest experimental measure of $T_{B} . T_{B}$ clearly increases with the MNP size as expected if one assumes that the energy barrier for the magnetization reversal, $\mathrm{E}_{\mathrm{B}}$, increases linearly with the particle volume $V$, i.e., $\mathrm{E}_{\mathrm{B}}=K_{\text {eff }} \mathrm{V}$, where $K_{\text {eff }}$ is the effective magnetic anisotropy constant. Moreover, coherently with the coercivity trend, $\mathrm{T}_{\mathrm{B}}$ increases from cubic $\left(\mathrm{C} 8 \_\mathrm{Hex}\right)$ to spherical (S8_Hex) shaped $\mathrm{MNPs}^{24}$ and from organic (n-hexane, S8_Hex) to polar (water, S8_Wat) solvent.

The spin blocking process has been studied also in the presence of a static applied field, by performing ZFC/FC measurements with applied fields in the range $\mu_{0} \mathrm{H}=0.005$ $-0.3 \mathrm{~T}$.

With the aim of obtaining for each sample the energy barrier distribution $\mathrm{E}_{\mathrm{B}}$ (average and width), we fitted the experimental data by using a model for non-interacting MNPs. ${ }^{27-29}$ It should be noticed that this model presents serious limits for the exact quantitative estimation of $\mathrm{E}_{\mathrm{B}}$, but allows us to grasp its general trend as a function of size, shape, and dispersant. ${ }^{32}$ On the other hand, no model able to predict the ZFC curve behavior of interacting MNPs is actually widely accepted in the literature, ${ }^{30}$ thus preventing us to use a more refined theory for the $\mathrm{E}_{\mathrm{B}}$ evaluation. The $\mathrm{ZFC}$ data were fitted by using the formula

$M_{Z F C}=\frac{\mu_{0} H M_{s}^{2}}{3 k_{B} K_{\text {eff }}^{2}}\left(\frac{1}{T} \int_{0}^{E_{\text {lim }}} E_{B}^{2} \rho\left(E_{B}\right) d E_{B}+\int_{E_{\text {lim }}}^{\infty} E_{B} \rho\left(E_{B}\right) d E_{B}\right)$,

where $\rho\left(E_{B}\right)=\frac{1}{E_{B} \sigma \sqrt{2 \pi}} e^{-\frac{\left(\ln E_{B}-\mu\right)^{2}}{2 \sigma^{2}}}$ is the log-normal distribution and $E_{\text {lim }}$ is the threshold energy for transition between the blocked state (particles with $E>E_{\text {lim }}$, ) and the unblocked SPM state (particles with $E<E_{\text {lim }}$ ). Figure 3 shows ZFC/FC magnetization curves recorded at $5 \mathrm{mT}$ for all samples Figs. 3(a)-3(c), ZFC/FC recorded at different fields for sample S8_Hex Fig. 3(d), and an example of fitting curve at $\mathrm{H}=5 \mathrm{mT}$ by means of Eq. (1) Fig. 3(e). The mean values of $E_{B}$ are reported in Figure 4 and in Table S1 of the supplementary material. As expected, $\mathrm{E}_{\mathrm{B}}$ monotonically decreases with increasing field. ${ }^{31}$

b. AC susceptibility. For the sake of comparison with the spin dynamics behavior detected by a microscopic probe (NMR) discussed in the next paragraph, we performed AC susceptibility measurements. To perform these measurements, the samples were dispersed in solution (hexane or water), as in NMR experiment, in order to reduce the interparticle interaction. An estimation of the dipole-dipole energy was obtained by the formula

$$
E_{d-d} \sim \frac{\mu_{0}}{4 \pi} \frac{\mu^{2}}{l^{3}},
$$

where $\mu=\mathrm{Ms} \mathrm{V}$ is the single particle magnetic moment and $l$ the interparticle distance. As can be seen from values reported
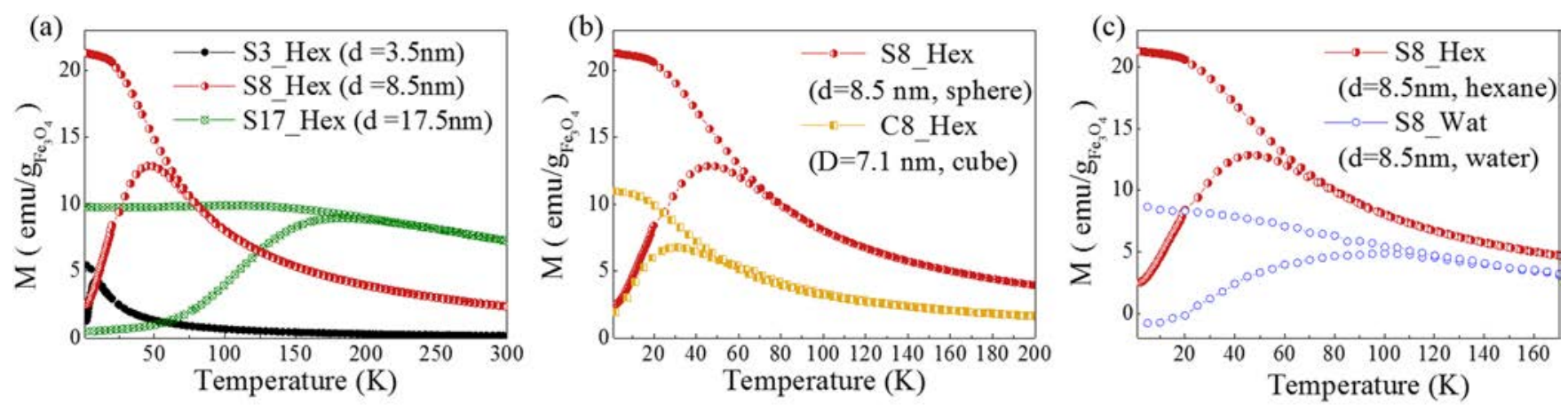

(d) $\quad$ S8_Hex
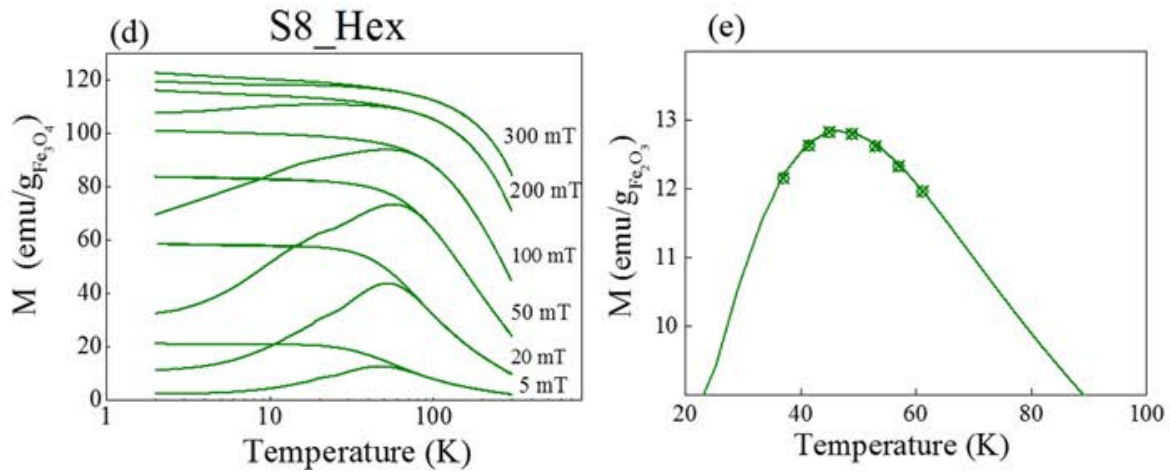

FIG. 3. ZFC/FC curves acquired with a $5 \mathrm{mT}$ probe field for samples with different (a) size, (b) shape, and (c) dispersant. (d) ZFC/FC curves of S8_Hex recorded applying different probe fields. (e) Experimental $\mathrm{ZFC}$ data (line) at $\mu_{0} \mathrm{H}=5 \mathrm{mT}$ compared with the best fit curve (dots) obtained in the range $35 \mathrm{~K}-65 \mathrm{~K}$. 


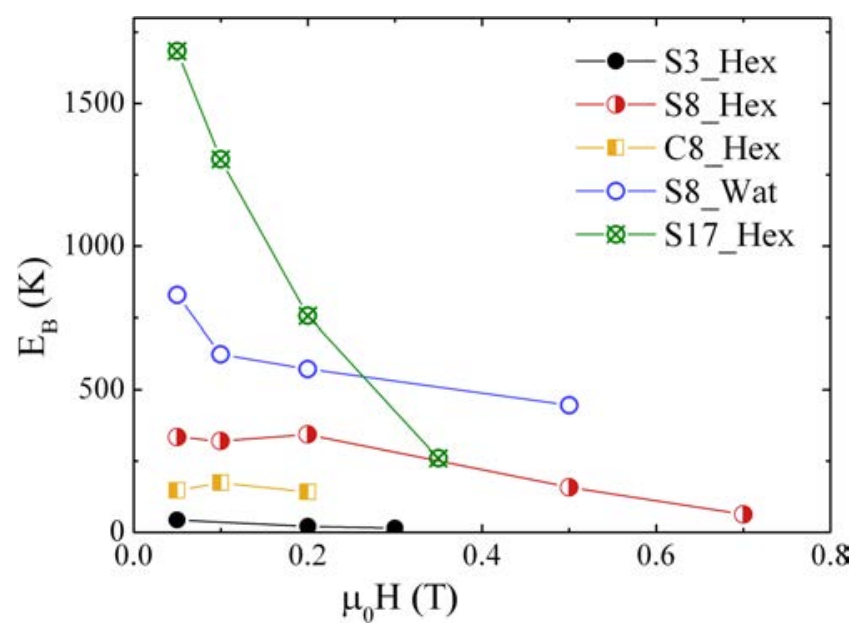

FIG. 4. Mean energy barrier calculated by fitting the ZFC curves to Eq. (1) for different fields.

in Table III, $E_{d-d}$ value is negligible for the smallest sample (S3_Hex) and for the water dispersed sample (S8_Wat), while for the other ones $E_{d-d} / k_{B} T>10^{3}$.

The in-phase $\chi^{\prime}(\omega, \mathrm{T})$ and out-of-phase $\chi^{\prime \prime}(\omega, \mathrm{T})$ components of $\chi$ of S8_Hex at different excitation frequencies are shown in Fig. 5 as representative of the behavior of all the samples. As the imaginary component $\chi^{\prime \prime}(\omega, \mathrm{T})$ is associated to the absorption of energy by the spin system due to the external alternating stimulus, its maximum for each $\omega_{\mathrm{AC}}$ occurs at $\mathrm{T}=\mathrm{T}_{\max }{ }^{\omega}$, when the typical correlation time of the magnetization $\tau_{\mathrm{N}}$ becomes equal to the inverse of the frequency of the external stimulus $\left(\tau_{\mathrm{N}} \cdot \omega_{\mathrm{AC}} \sim 1\right)$.

In order to evaluate the crucial spin dynamics parameters $\left(\tau_{0}, T_{0}\right.$, and $\left.E_{B}\right)$, the set of experimental points $\tau_{\mathrm{N}} \mathrm{vs} 1 / \mathrm{T}_{\max }{ }^{\omega}$ have been fitted to the VF model, $\tau=\tau_{0} e^{\frac{E_{B}}{k_{B}\left(T-T_{0}\right)}}$.

As the presence of three parameters can generate nonconverging fits, we used the $\mathrm{E}_{\mathrm{B}}$ value estimated from low field DC measurements, as a rough approximation that allowed us to have just two remaining free fitting parameters. The values of $\tau_{0}, T_{0}$, and $E_{B}$ obtained from the AC data analysis, reported in Table III, are in the typical range for SPM systems for all the investigated samples (due to the synthesis conditions, S8_Wat is very diluted and AC data could not be reproducibly collected).

As expected, the value of $T_{0}$ follows the dipole-dipole interaction energy trend. In particular $T_{0}$ and $E_{d-d}$ increase with the size of the MNPs (from S3_Hex to S8_Hex to S17_Hex) and from spherical (S8_Hex) to cubic-shaped

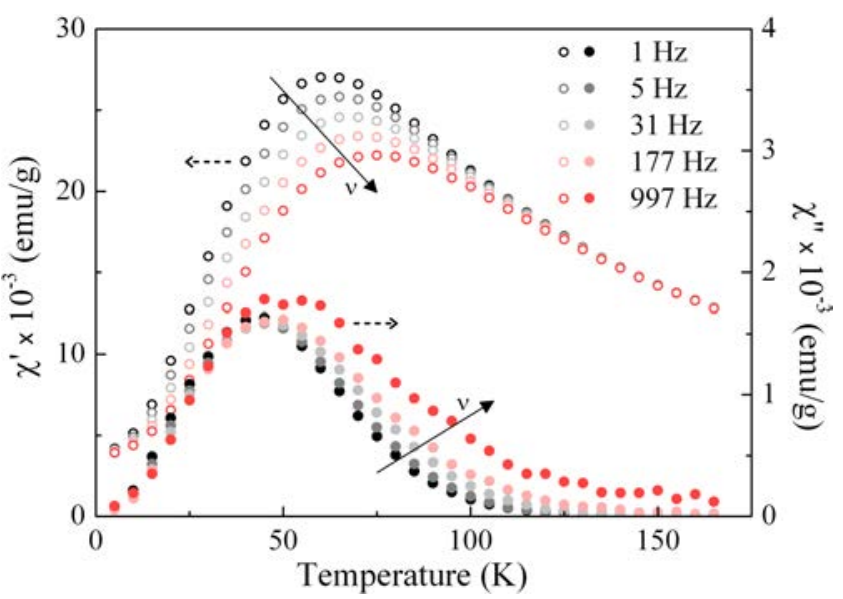

FIG. 5. Temperature dependence of the in-phase $\chi^{\prime}(\mathrm{T})$ (empty circles) and out-of-phase $\chi^{\prime \prime}(\mathrm{T})$ components (solid circles) of the magnetic susceptibility, with excitation frequencies in the $1-1000 \mathrm{~Hz}$ range for sample S8_Hex. Arrows indicate the increasing frequencies.

MNPs (C8_Hex).It should be noticed that the high $\mathrm{T}_{0}$ for S17_Hex is associated to the high magnetic moment determined by its large volume that compensates the low value of $M_{s}$ due to the presence of wustite in the sample.

\section{C. ${ }^{1} \mathrm{H}-\mathrm{NMR}$ dispersion profiles}

${ }^{1}$ H-NMRD profiles have been collected in a wide frequency range, selected to cover the frequencies associated to the fields used by most common clinical imagers, i.e., $\mu_{0} \mathrm{H}=0.2,0.5$, and $1.5 \mathrm{~T}$ (corresponding to $\sim 8,20$, and $64 \mathrm{MHz}$ ) and to allow the investigation of the physical mechanisms responsible for nuclear relaxation through the analysis of the $r_{1}(v)$ and $r_{2}(v)$ curves. To obtain the NMR-D profiles, we have calculated the nuclear relaxivities, defined as

$$
r_{i}=\left[\left(1 / T_{i}\right)_{\text {meas }}-\left(1 / T_{i}\right)_{\text {dia }}\right] / c \quad i=1,2,
$$

where $\left(1 / T_{i}\right)_{\text {meas }}$ is the value measured for the sample with density of magnetic center $c\left(\mathrm{mmol} \mathrm{l}^{-1}\right)$ and $\left(1 / T_{i}\right)_{\text {dia }}$ represents the nuclear relaxation rate of the diamagnetic host solution (hexane or water in our case). In Fig. 6, we report the experimental data, where the effect of size, shape, and dispersant on longitudinal and transverse relaxivity is outlined. The longitudinal relaxivity $\mathrm{r}_{1}$ follows the typical behavior of superparamagnetic MNPs, displaying a plateau at low frequencies, a pronounced maximum, and finally a decrease at higher frequencies. The maximum of the $r_{1}$ curve displaces toward higher frequencies by reducing the size of the MNPs, as shown

TABLE III. From the left: $\mathrm{E}_{\mathrm{B}}$ and $\sigma \mathrm{E}_{\mathrm{B}}$ are the mean value and standard deviation of the energy barrier lognormal distribution as obtained from the fitting of ZFC curves to Equation (1); the VF law parameters $\tau_{0}$ and $\mathrm{T}_{0}$ as obtained from the AC data analysis (see text); the particle concentration and the estimated dipole-dipole energy at room temperature.

\begin{tabular}{|c|c|c|c|c|c|c|c|}
\hline Sample $5 \mathrm{mT}$ & $\mathrm{E}_{\mathrm{B}}(\mathrm{K})$ & $\sigma \mathrm{E}_{\mathrm{B}}(\mathrm{K})$ & $\tau_{0}(s)$ & $\mathrm{T}_{0}(\mathrm{~K})$ & $\tau_{\mathrm{N}} \mathrm{AC}, 300 \mathrm{~K}(\mathrm{~s})$ & $\frac{V_{N P s}}{V}$ & $\frac{E_{d-d}}{k_{B} T}$ \\
\hline S3_Hex & 43 & 38 & $(5.5 \pm 1.1) \times 10^{-8}$ & $4.15 \pm 0.5$ & $(4.7 \pm 1.3) \times 10^{-8}$ & $5.5 \times 10^{-5}$ & 6.7 \\
\hline S8_Hex & 334 & 194 & $(2.4 \pm 0.8) \times 10^{-11}$ & $30.5 \pm 2.3$ & $(6.9 \pm 1.2) \times 10^{-10}$ & $4.4 \times 10^{-4}$ & 2000 \\
\hline C8_Hex & 146 & 145 & $(1.5 \pm 0.9) \times 10^{-9}$ & $18.2 \pm 1.1$ & $(8.8 \pm 2.6) \times 10^{-10}$ & $6.9 \times 10^{-4}$ & 7000 \\
\hline S8_Wat & 829 & 442 & $\ldots$ & $\ldots$ & $\ldots$ & $4.2 \times 10^{-6}$ & 22 \\
\hline S17_Hex & 1683 & 711 & $(4.4 \pm 2.1) \times 10^{-14}$ & $160 \pm 8.6$ & $(7.3 \pm 3.1) \times 10^{-9}$ & $3.4 \times 10^{-4}$ & 9000 \\
\hline
\end{tabular}




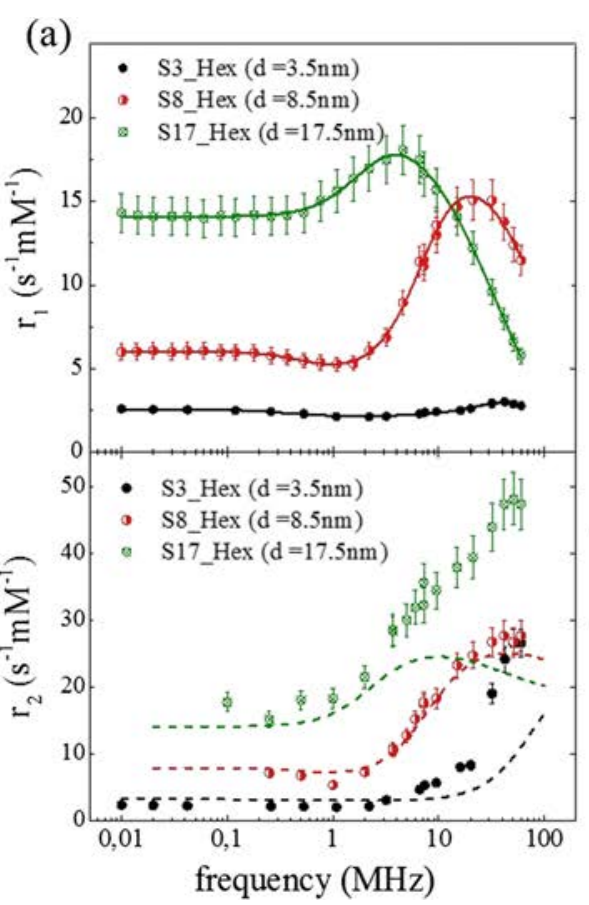

(b)

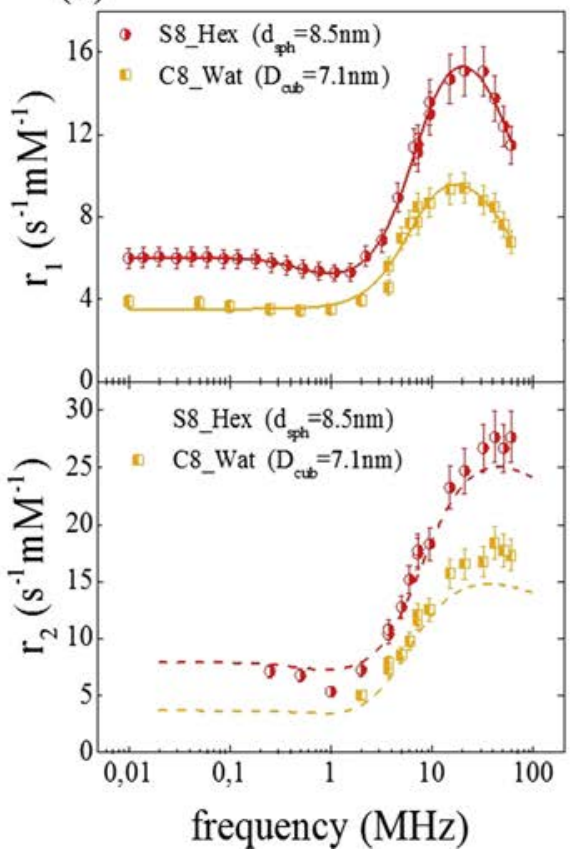

(c)

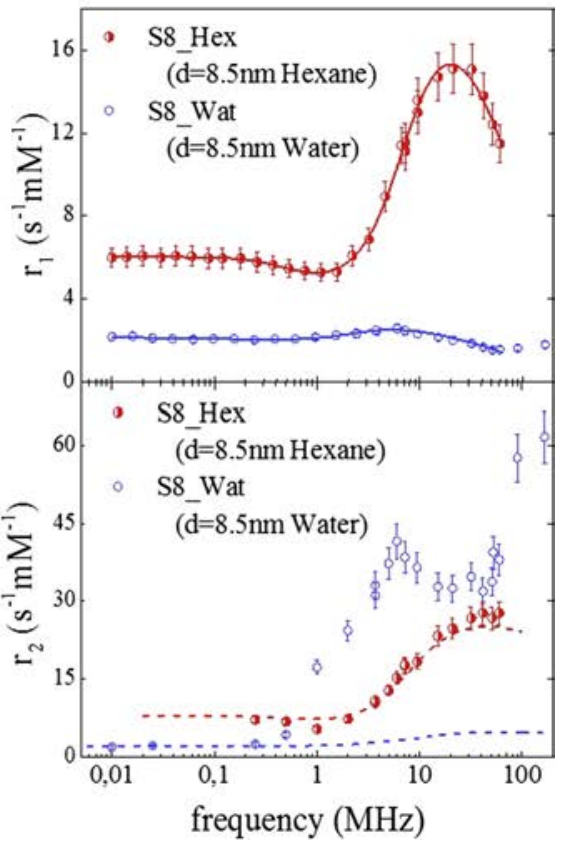

FIG. 6. Longitudinal $\left(\mathrm{r}_{1}\right)$ and transverse $\left(\mathrm{r}_{2}\right)$ relaxivities (NMRD profiles) at room temperature in the frequency range $0.01<v<120 \mathrm{MHz}$ measured on samples with different size (a), shape (b), and dispersant (c). The solid lines represent the best-fit curves of $\mathrm{r}_{1}$ obtained from the Roch's model (see Equation (2)). The dashed lines are the $r_{2}$ calculation performed using Eq. (3) and the best fit parameters obtained for $r_{1}$.

in Figure 6(a). An indication about the value of the magnetic anisotropy can be obtained looking at the so-called "low field dispersion," i.e., the slight dip just after the low frequency plateau. In our case, the dispersion feature becomes less evident with increasing the size from S3_Hex to S17_Hex.

The cubic shaped sample C8_Hex (Figure 6(b)) and the water dispersed sample S8_Wat (Figure 6(c)) display a lower longitudinal relaxivity over the whole frequency range with respect to the spherical hexane dispersed sample S8_Hex.

To fit the NMR relaxivity profiles, we used the heuristic model by Roch et al. ${ }^{14}$ To understand the physical background of the model, one should first observe that magnetic dispersed nanoparticles create local field inhomogeneities which modify the nuclear relaxation process of the dispersant protons with respect to the ones in the pure solvent. Thus, the equations of the relaxivity must describe the nuclear relaxation induced by the fluctuating hyperfine interaction between the particle magnetic moment and the nuclear magnetic moment of the hydrogen nuclei of the solvent. A first contribution to the nuclear relaxation arises from the hyperfine field fluctuations at the nuclear sites caused by the diffusion of solvent protons into the inhomogeneous magnetic field created by the large magnetic moments of the MNP (Curie relaxation). A second contribution comes from the fluctuations of the magnetic moment of each particle induced by the Néel relaxation with typical correlation time $\tau_{N}$.

Despite the author calculated the exact expression for the relaxation rates, in order to overcome the computational time constrains, a heuristic model was alternatively proposed by the same authors. ${ }^{13,14}$ In their model, they linearly combined the relaxivity equation describing the null magnetic anisotropy case with the one describing the infinite magnetic anisotropy system. The corresponding heuristic expressions for $r_{1}$ and $r_{2}$ are

$$
\begin{aligned}
r_{1}= & \frac{32 \pi}{135000} \mu_{S P}^{* 2} \gamma_{I}^{2}\left(\frac{N_{a} c}{r_{d} D}\right) x\left\{7 P \frac{L(x)}{x} J^{F}\left(\omega_{s}, \tau_{D}, \tau_{N}\right)\right. \\
& +\left[7 Q \frac{L(x)}{x}+3(P+Q)\left(1-L^{2}(x) \mp 2 \frac{L(x)}{x}\right)\right] \\
& \left.\times x J^{F}\left(\omega_{I}, \tau_{D}, \tau_{N}\right)+3 L^{2}(x) J^{A}\left(\sqrt{2 \omega_{I} \tau_{D}}\right)\right\} \\
r_{2}= & \frac{16 \pi}{135000} \mu_{S P}^{* 2} \gamma_{I}^{2}\left(\frac{N_{a} c}{r_{d} D}\right) x\left\{13 P \frac{L(x)}{x} J^{F}\left(\omega_{s}, \tau_{D}, \tau_{N}\right)\right. \\
+ & 7 Q \frac{L(x)}{x} J^{F}\left(\omega_{I}, \tau_{D}, \tau_{N}\right)+6 Q \frac{L(x)}{x} J^{F}\left(0, \tau_{D}, \tau_{N}\right) \\
+ & \left(1-L^{2}(x)-2 \frac{L(x)}{x}\right) x\left[3 J^{F}\left(\omega_{I}, \tau_{D}, \tau_{N}\right)\right. \\
+ & \left.\left.4 J^{F}\left(0, \tau_{D}, \tau_{N}\right)\right]+L^{2}(x)\left[3 J^{A}\left(\sqrt{2 \omega_{H} \tau_{D}}\right)+4 J^{A}(0)\right]\right\}
\end{aligned}
$$

where $\mathrm{P}$ and $\mathrm{Q}$ are weighting factors and the constraint $\mathrm{P}+\mathrm{Q}=1$ is valid. The two limit cases are $\mathrm{P}=1$ for $\mathrm{E}_{\mathrm{A}}=0$ and $\mathrm{Q}=1$ for $\mathrm{E}_{\mathrm{A}} \rightarrow \infty$. Additionally, $\mu_{S P}^{*}=A \mu_{S P}$ is the effective magnetic moment felt by the protons locally (the "local probe"), $\gamma_{I}$ is the proton gyromagnetic ratio, $r$ is the minimum approach distance of the solvent protons to the MNPs, $\mathrm{D}$ is the self-diffusion coefficient of the medium $\left(1.92 \times 10^{-9}\right.$ $\mathrm{m}^{2} \mathrm{~s}^{-1}$ for water and $4.26 \times 10^{-9} \mathrm{~m}^{2} \mathrm{~s}^{-1}$ for $\mathrm{n}$-hexane), $\mathrm{N}_{\mathrm{a}}$ is the Avogadro number, $\mathrm{c}$ is the molar concentration of nanoparticles, $\mathrm{L}(\mathrm{x})$ is the Langevin function where $x=\mu_{S P} B_{0} / k_{B} T$, $\tau_{\mathrm{D}}$ and $\tau_{\mathrm{N}}$ are the diffusion and Néel time at room temperature, respectively, $\omega_{\mathrm{S}}$ and $\omega_{\mathrm{I}}$ are the electron and proton 
resonance frequencies, and $\omega_{0}$ is a free parameter that adjusts the low frequency behavior. Eqs. (2) and (3) are the results of the proper weight of the low field contribution described by the Freed function,

$$
J^{F}\left(\omega, \tau_{D}, \tau_{N}\right)=\mathfrak{R}\left[\frac{1+\sqrt{\Omega} / 4}{1-\sqrt{\Omega}+4 \Omega / 9+\sqrt{\Omega^{3}} / 9}\right],
$$

where $\Omega=\left(i \omega+1 / \tau_{N}\right) \tau_{D}$, and the high field contribution given by the Ayant spectral density function ${ }^{34}$

$$
J^{A}(z)=\frac{1+5 z / 8+z^{2} / 8}{1+z+z^{2} / 2+z^{3} / 6+4 z^{4} / 81+z^{5} / 81+z^{6} / 648},
$$

with $z=\sqrt{2 \omega_{I} \tau_{D}}$.

The $\mathrm{r}_{1}$ fitting procedures of the NMR-D profiles with Eq. (2) required $\mu_{S P}^{*}, \mathrm{P}, \mathrm{Q}, \mathrm{r}$, and $\tau_{\mathrm{N}, \mathrm{NMR}}$ as free fitting parameters. Table IV reports the fitting values for the free parameters. The distance $r$ has been used to calculate the value of $\langle d\rangle_{N M R}^{H}=2 r$ that could be compared to the hydrodynamic size calculated from DLS measurements. As it can be seen in Table IV $\langle d\rangle_{N M R}^{H}$ and $\langle d\rangle_{D L S}^{H}$ have different values reflecting the fact that the DLS technique overestimates the distance of minimum approach; moreover the DLS measurements are less reliable for small particles such as the ones presented here.

The P/Q ratio, as expected, increases with decreasing anisotropy and displays a trend similar to that of the anisotropy energy barrier (see Table III).

The values of the Néel relaxation times at room temperature, $\tau_{\mathrm{N}}{ }^{\mathrm{NMR}}$, are in the typical range of SPM compounds reversal time, i.e., $10^{-10}-10^{-7} \mathrm{~s}$. By considering that the higher the anisotropy barrier, the slower the relaxation time, sample S17_Hex, which displays the highest energy barrier among all the samples in hexane, shows the slowest $\tau_{N}{ }^{N M R}$. The relaxation time, $\tau_{N}{ }^{N M R}$ follows the $E_{B}$ trend also for the two $8.5 \mathrm{~nm}$ samples, becoming smaller from S8_Hex to C8_Hex (from spherical to cubic shape) and higher from S8_Hex to S8_Wat (from polar to non polar solvent).

With the aim of comparing the value of the Néel relaxation time at room temperature estimated from NMR-D profiles and $\mathrm{AC}$ susceptibility data, in Fig. 7 we report the ratio $\tau_{\mathrm{N}, \mathrm{NMR}} / \tau_{\mathrm{N}, \mathrm{AC}}$

TABLE IV. Fitting parameters obtained from the analysis of ${ }^{1} \mathrm{H}-\mathrm{NMR}-\mathrm{D} \mathrm{r}_{1}$ profiles employing Roch's model, Eqs. (2) and (3). The last two columns refer to the distance (multiplied by a factor 2) of minimum approach between the protons of the solution and the MNP centers obtained by NMR and to the hydrodynamic diameter extracted from DLS measurements, respectively. The polydispersity index for DLS measurements was found to be less than 0.2 for all the hexane dispersions and 0.4 for the water-based sample (S8_Hex).

\begin{tabular}{lcccccc}
\hline \hline Sample & $\mu_{S P}^{*} / \mu$ & P/Q & $\begin{array}{c}\tau_{\mathrm{N}}{ }^{\mathrm{NMR}, 300 \mathrm{~K}} \\
(\mathrm{~s})\end{array}$ & $\begin{array}{c}\mathrm{e}_{2} / \mathrm{r}_{1} \\
(60 \mathrm{MHz})\end{array}$ & $\begin{array}{c}\langle\mathrm{d}\rangle_{\mathrm{NMR}}^{\mathrm{H}} \\
(\mathrm{nm})\end{array}$ & $\begin{array}{c}\langle\mathrm{d}\rangle_{\text {DLS }}^{\mathrm{H}} \\
(\mathrm{nm})\end{array}$ \\
\hline S3_Hex & 1 & 0.29 & $1.8 \times 10^{-9}$ & 9.6 & $17.6 \pm 4.5$ & n.a. \\
S8_Hex & 0.96 & 0.51 & $8.7 \times 10^{-10}$ & 2.4 & $10.4 \pm 0.5$ & 12.5 \\
C8_Hex & 0.67 & 0.59 & $6.7 \times 10^{-10}$ & 2.6 & $10.9 \pm 0.7$ & 20.4 \\
S8_Wat & 0.96 & 0.09 & $9.9 \times 10^{-9}$ & 24.5 & $14.5 \pm 0.9$ & 50.0 \\
S17_Hex & 0.33 & 0.05 & $9.4 \times 10^{-9}$ & 8.2 & $20.1 \pm 1.5$ & 29.5 \\
\hline \hline
\end{tabular}

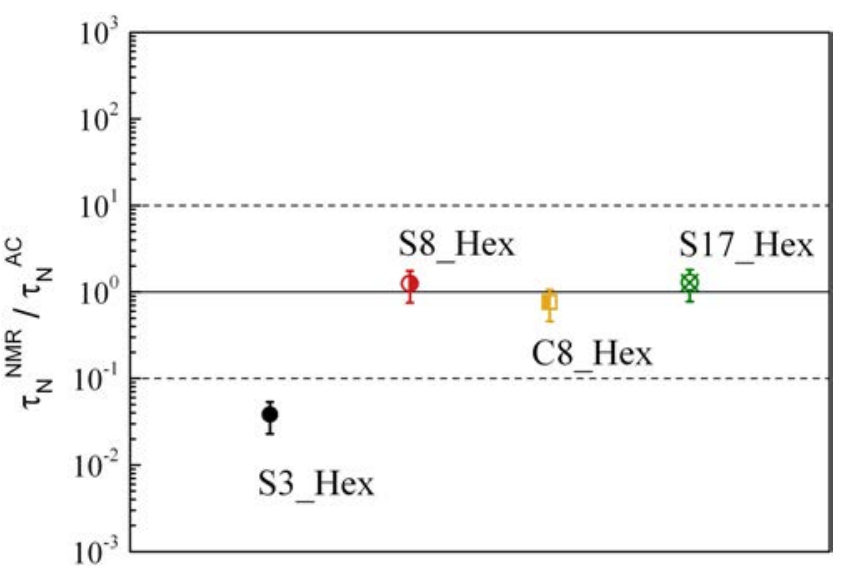

FIG. 7. $\tau_{\mathrm{N}}^{\mathrm{NMR}} / \tau_{\mathrm{N}}^{\mathrm{AC}}$ ratios for all investigated samples. The two dashed lines delimit the range of tolerance of one order of magnitude.

It should be stressed that the $\mathrm{AC}$ susceptibility technique provides results averaged over macroscopic dimensions, while NMR uses a local probe (the nucleus) and so is less sensitive to the effect of long range dipolar interparticle interactions. Nonetheless, the values of $\tau_{\mathrm{N}, \mathrm{NMR}}$ display a good agreement with $\tau_{\mathrm{N}, \mathrm{AC}}$ except for the smallest sample S3_Hex.

The discrepancy observed for this sample is probably due to its high surface to volume ratio. Indeed, the high number of surface spins with respect to the core ones can influence the interaction between the protons of the solvent passing near the MNP surface, leading to significant differences in the spin dynamics as seen by NMR (local probe) and AC magnetometry (bulk probe).

In the framework of biomedical applications for MNPs, NMR-D curves are suitable to predict the efficiency as negative CAs in magnetic resonance imaging (MRI). The most important parameter for negative CAs is the increase of the $\mathrm{T}_{2}$-relaxation per millimole of magnetic center. ${ }^{9,13}$ Fig. 6(a) suggests that in the frequency range of clinical use (e.g., for magnets operating at about $v=8 \mathrm{MHz}, 20 \mathrm{MHz}$, or 60 $\mathrm{MHz}$ ) sample S17_Hex has the highest transverse relaxivity among the hexane samples $\left(\sim 50 \mathrm{mM}^{-1} \mathrm{~s}^{-1}\right.$ at 60 $\mathrm{MHz}$ ). It is noteworthy, however, that the sample S3_Hex for relatively high frequencies $(v>40 \mathrm{MHz})$ displays $r_{2}$ values sufficiently high to give an enough good contrast $\left(\sim 20 \mathrm{mM}^{-1} \mathrm{~s}^{-1}\right.$ at $\left.60 \mathrm{MHz}\right)$ despite its very small size. The latter result is of certain relevance especially as concerns in vivo administration, where MNPs of few nanometers are largely preferred. ${ }^{35}$ Intravenous administration of contrast agents also requires MNPs to be dispersible in aqueous solutions. In this sense, Fig. 6(c) shows the role of the solvent on relaxation times: the transfer into a water-based dispersant (sample S8_Wat) increases the efficiency (i.e., $r_{2}$ ) of the MNPs as compared to the same sample dispersed in hexane (sample S8_Hex).

Thus, our systems are more efficient in aqueous solution as required for in vivo applications.

Regarding the $r_{2}$, it should be noticed that the experimental values increase with the size of magnetic core, decrease from spherical to cubic shaped sample, and increase when the solvent is changed from hexane to water. A high field double maximum feature not predicted by the theory also appears in 


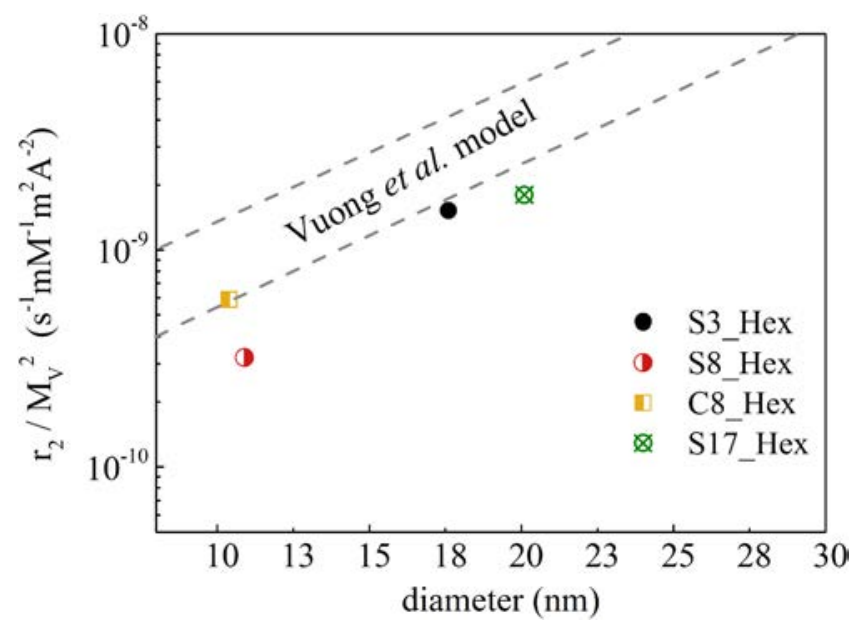

FIG. 8. Influence of the size on transverse relaxivity at high field $(60 \mathrm{MHz})$ for sample in the motional averaging regime (MAR). The grey zone indicates the region of validity of the model ${ }^{16}$ by Vuong et al.

sample S8_Wat. By fitting the complete profile with Eq. (3), it seems that the experimental data show some discrepancies with the theoretical ones (Fig. 6), obtained by using the same fit parameters values $\mu_{S P}^{*}, \mathrm{P}, \mathrm{Q}, \mathrm{r}$, and $\tau_{\mathrm{N}, \mathrm{NMR}}$ used for $\mathrm{r}_{1}$ fitting. In particular, it should be noted that the $r_{2}$ theoretical curves do not reproduce experimental data in the high field region, being lower than experimental ones. We suggest that one or more unknown physical mechanisms (i.e., interaction between MNPs, chemical exchange with the water molecule of the bulk, dipole-dipole nuclear interaction, etc), not included in the Roch's model, contribute to $r_{2}$ in the high field regime. ${ }^{33}$

Finally, we compared the $r_{2}$ experimental behavior with a universal scaling law ${ }^{16}$ recently proposed for systems in the so-called "motional averaging regime" (MAR) ${ }^{36,37}$ where $\omega \tau_{c} \ll 1\left(\tau_{c}\right.$ being the dominating correlation time). Vuong et al. demonstrated that a quadratic dependence of $\mathrm{r}_{2}$ on the particle hydrodynamic diameter occurs at high frequencies

$$
\frac{r_{2}}{M_{V}^{2}}=11.6 \times 10^{-12} d_{H}^{2}
$$

All of our samples are in the MAR regime at $v=60 \mathrm{MHz}$; in Table $S \_2$ of the supplementary material the main parameters used in Eq. (4) are reported. Figure 8 shows that the transverse $r_{2}$ relaxivity of most of our samples (data at $60 \mathrm{MHz}$ ) falls very near to the ones predicted by the scaling law, thus giving a good approximation for $r_{2}$ expression at high fields, where the Roch model fails.

\section{CONCLUSIONS}

We investigated the spin dynamics of iron oxide colloidal suspensions of MNPs capped by oleic acid with variable core diameter $(\mathrm{d}=3.5,8.5$, and $17.5 \mathrm{~nm})$, shape (spherical and cubic), and dispersant (hexane or water). The magnetic properties have been investigated by means of DC and AC magnetic techniques, from which the saturation magnetization $M_{S}$, the coercive field $H_{c}$, the blocking temperature, $T_{B}$, and the anisotropy barrier, $\mathrm{E}_{\mathrm{B}}$, distribution have been estimated. Values of $H_{c}$ and $M_{s}$ are found to increase with the particle size (the low value of saturation magnetization displayed by
S17_Hex is ascribed to the presence of wustite) and to decrease from spherical to cubic shape.

In order to study the fundamental physical mechanisms of spin dynamics that accelerate the nuclear relaxation, the NMR-D curves for the longitudinal $\left(r_{1}\right)$ and transverse $\left(r_{2}\right)$ relaxivities were recorded over the frequency range $0.01 / 120$ MHz. The $r_{1}(v)$ profiles have been successfully fitted with the model of superparamagnetic particles by Roch et al. ${ }^{14}$ The distance of minimum approach extracted from the fits is in good agreement with the hydrodynamic diameters measured by DLS data. The reversal time of magnetization $\left(\tau_{\mathrm{N}}\right)$ estimated by NMR experiments is in good agreement with the ones obtained from AC susceptibility ones, except for sample S3_Hex, where the small diameter influences differently the local and the bulk spin dynamics.

The $r_{2}$ profiles could not be fitted by using the parameter values obtained from $r_{1}$ fitting. This disagreement is thought to be due to further physical mechanisms contributing to the nuclear relaxation (water exchange, interparticle interactions, etc. ${ }^{33}$ ) at high fields, not taken into account by the Roch's model. On the other hand, the approximate $r_{2}$ model by Vuong et al. ${ }^{16}$ has been shown a rough agreement with our results.

\section{SUPPLEMENTARY MATERIAL}

See supplementary material for list of the main magnetic parameters as a function of field; lists of parameters used to test Vuong model nanoparticle in the MAR regime; parameter $\mathrm{T}_{0}$ and $\mathrm{t} 0$ as obtained by fitting AC data by the VF law. XRay diffraction patterns for the MNPs and additional TEMs of sample S8_Hex.

\section{ACKNOWLEDGMENTS}

The COST-RADIOMAGN from EU, Grant No. ID1402, the Italian projects INSTM-Regione Lombardia "MagNANO," FIRB "Riname," and Fondazione Banco di Sardegna are acknowledged for financial support. Daniela Maggioni, Andrea Barbaglia, and Matteo Castare are gratefully acknowledged for help in experimental measurements and data analysis.

${ }^{1}$ S. Karmakar et al., J. Phys.: Conf. Ser. 292, 012002 (2011).

${ }^{2}$ C. Chappert et al., Nat. Mater. 6, 813 (2007).

${ }^{3}$ B. D. Terris and T. Thomson, J. Phys. D: Appl. Phys. 38, R199 (2005).

${ }^{4}$ A. Lascialfari and C. Sangregorio, Chem. Today 29, 20-23 (2011).

${ }^{5}$ R. Hergt et al., J. Phys.: Condens. Matter 20, 385214 (2008).

${ }^{6}$ The Chemistry of Contrast Agents in Medical Magnetic Resonance Imaging, edited by A. E. Merbach and E. Toth (Wiley, New York, 2001).

${ }^{7}$ A. Lascialfari et al., Riv. Nuovo Cimento 36(6), 211 (2013).

${ }^{8}$ V. K. Varadan, L. F. Chen, and J. Xie, Nanomedicine: Design and Applications of Magnetic Nanomaterials, Nanosensors and Nanosystems (Wiley, 2008).

${ }^{9}$ D. Kunk et al., J. Chem. Phys. 140, 174504 (2014).

${ }^{10}$ C. Cannas et al., J. Chem. Phys. 125, 164714 (2006).

${ }^{11}$ R. K. Dani et al., J. Chem. Phys. 135, 224502 (2011).

${ }^{12}$ A. Robert et al., J. Chem. Phys. 122, 084701 (2005).

${ }^{13}$ E. W. C. Limand and R. Feng, J. Chem. Phys. 136, 124109 (2012).

${ }^{14}$ A. Roch et al., J. Chem. Phys. 110, 5403 (1999).

${ }^{15}$ P. Gills, A. Roch, and R. A. Brooks, J. Magn. Reson. 137, 402 (1999).

${ }^{16}$ Q. L. Vuong et al., Adv. Healthcare Mater. 1, 502 (2012).

${ }^{17}$ P. Guardia et al., Chem. Commun. 46, 6108 (2010).

${ }^{18}$ P. Guardia et al., ACS Nano 6(4), 3080 (2012). 
${ }^{19}$ P. Guardia et al., J. Phys. Chem. C 115(2), 390 (2011).

${ }^{20}$ S. Murgia et al., J. Phys. Chem. B 114(10), 3518 (2010).

${ }^{21}$ M. Carboni et al., Adv. Healthcare Mater. 2, 692 (2013).

${ }^{22}$ R. Angelico et al., Soft Matter 9, 921 (2013).

${ }^{23}$ L. Bordonali et al., J. Phys.: Condens. Matter 25, 066008 (2013).

${ }^{24}$ G. Salarez-Alvarez et al., J. Am. Chem. Soc. 130, 13234 (2008).

${ }^{25}$ H. Vogel, Phys. Z. 22, 645 (1921); G. S. Fulcher, J. Am. Ceram. Soc. 8, 339 (1925).

${ }^{26}$ Z. Zhang and S. Satpathy, Phys. Rev. B 44(24), 13319 (1991).

${ }^{27}$ F. Tournus and E. Bonet, J. Magn. Magn. Mater. 323, 1109 (2011).

${ }^{28}$ F. Tournus and A. Tamion, J. Magn. Magn. Mater. 323, 1118 (2011).

${ }^{29}$ L. Bordonali, "Novel magnetic nanoparticles for MRI: Dependence of nuclear relaxation on microscopic parameters," Ph.D. thesis, Università degli Studi Roma TRE e Consorzio Nazionale Interuniversitario per le Scienze Fisiche della Materia, 2012.

${ }^{30}$ B. Aslibeiki, P. Kameli, and H. Salamati, J. Appl. Phys. 119, 063901 (2016).

${ }^{31}$ X. Battle and A. Labarta, J. Phys. D: Appl. Phys. 35(6), R15 (2002).

${ }^{32}$ T. Orlando et al., J. Appl. Phys. 119, 134301 (2016).

${ }^{33}$ Y. Gossuin et al., Nanotechnology 27, 155706 (2016).

${ }^{34}$ Y. Ayant et al., J. Phys. 36, 991 (1975).

${ }^{35}$ N. Singh et al., Nano Rev. 1, 5358 (2010).

${ }^{36}$ R. A. Brooks, F. Moiny, and P. Gills, Magn. Reson. Med. 45, 1014 (2001).

${ }^{37}$ P. Gills, F. Moiny, and R. A. Brooks, Magn. Reson. Med. 263(2), 1014 (2002). 